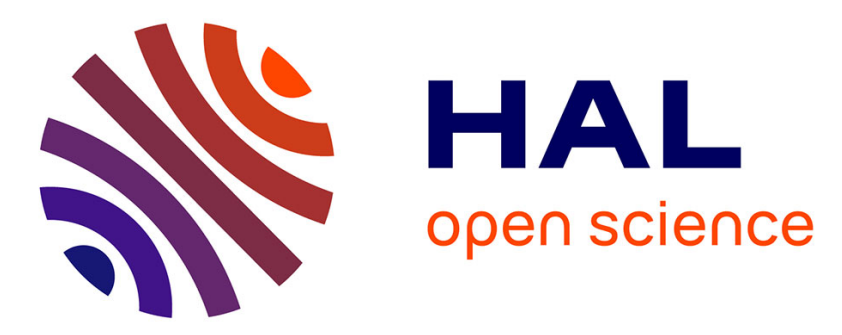

\title{
Optical properties of quantum wells with a field-effect-induced lateral superlattice
}

\author{
C. Peters, W. Hansen, J. Kotthaus, M. Holland
}

\section{To cite this version:}

C. Peters, W. Hansen, J. Kotthaus, M. Holland. Optical properties of quantum wells with a field-effectinduced lateral superlattice. Journal de Physique IV Proceedings, 1993, 03 (C5), pp.C5-123-C5-130. 10.1051/jp4:1993522 . jpa-00251608

\section{HAL Id: jpa-00251608 https://hal.science/jpa-00251608}

Submitted on 1 Jan 1993

HAL is a multi-disciplinary open access archive for the deposit and dissemination of scientific research documents, whether they are published or not. The documents may come from teaching and research institutions in France or abroad, or from public or private research centers.
L'archive ouverte pluridisciplinaire HAL, est destinée au dépôt et à la diffusion de documents scientifiques de niveau recherche, publiés ou non, émanant des établissements d'enseignement et de recherche français ou étrangers, des laboratoires publics ou privés. 


\title{
Optical properties of quantum wells with a field-effect-induced lateral superlattice
}

\author{
C. PETERS, W. HANSEN, J.P. KOTTHAUS and M. HOLLAND*
}

Sektion Physik, Ludwig-Maximilians-Universität, Geschwister-Scholl-Platz 1, 80539 München, Germany ${ }^{*}$ DEEE, University of Glasgow, $128 Q Q, U . K$.

\begin{abstract}
A new developed stacked gate technique and a special MBE-grown quantum well structure is used to investigate the optical properties of a quantum well in a field effect induced lateral superlattice potential. Gate voltages applied at two different gates allow to tune both the superlattice potential amplitude as well as the energetical position with respect to the Fermienergy. The luminescence and luminescence excitation spectra show a strong dependence on these voltages. At certain gate voltage configurations electrons are filled into the minima of the lateral potential and form an array of quasi one-dimensional electron channels. At this point the luminescence splits into distinct peaks reflecting the lateral separation of photo excited electrons and holes. Excitation measurements illustrate the existence of occupied electron channels and unoccupied barriers between them.
\end{abstract}

\section{Introduction}

Presently there is great interest in the optical properties of one- and zero-dimensional electron systems. For optical investigations these are prepared by patterning a two-dimensional electron gas (2DEG) in a GaAs/AlGaAs quantum well system. Pattering tools are various etching techniques [1][2][3], laser induced interdiffusion [4], special growth techniques [5], or stress inducing masks on the top of the sample [6]. There is no direct way to tune the lateral confinement potential in these samples after the initial preparation of the one- or zero-dimensional structure. In contrast to the previously described techniques field-effect-induced lateral potentials can be tuned during the measurement. So far there are no optical investigations of field effect induced one- or zero-dimensional structures because the lateral gate structure samples used for far-infrared (FIR) spectroscopy [7] or transport measurements [8] are not functional under illumination with light of an energy greater then the bandgap of GaAs. However, there are many observations of the influence of vertical electric fields on the optical properties of GaAs/AlGaAs quantum well structures [9][10][11]. Here we report on optical investigations of fieldeffect-induced lateral superlattices at the location of a GaAs quantum well (QW) imbedded in a MBE grown heterostructure designed to be suitable for field-effect-tuning under optical illumination. With luminescence (PL) and excitation (PLE) studies we demonstrate that these devices can be electrically tuned from a 2D laterally homogenous quantum well to $1 \mathrm{D}$ quantum well wires. 


\section{Laterally homogenous quantum well devices}

For our studies we have developed a special molecular-beam-epitaxy grown heterostructure with a $15 \mathrm{~nm}$ wide GaAs QW between barriers consisting of a GaAs/AlAs superlattice (SL). Here a GaAs/AlAs SL is used as barrier because its resistance under illumination is found to be higher than the resistance of a AlGaAs alloy. The sketch of the bandstructure in Fig. 1 illustrates that there is no doping in the vicinity of the quantum well close to and especially in the barrier between the front gate and the QW. This avoids the formation of a light-induced conducting bypass between the $\mathrm{QW}$ and the surface of the sample which otherwise screens any lateral potential modulation effectively. With voltage $V_{g}$ applied between a gate on the sample surface and a heavily doped back contact $300 \mathrm{~nm}$ beneath the quantum well we can control the electric field across of the quantum well.

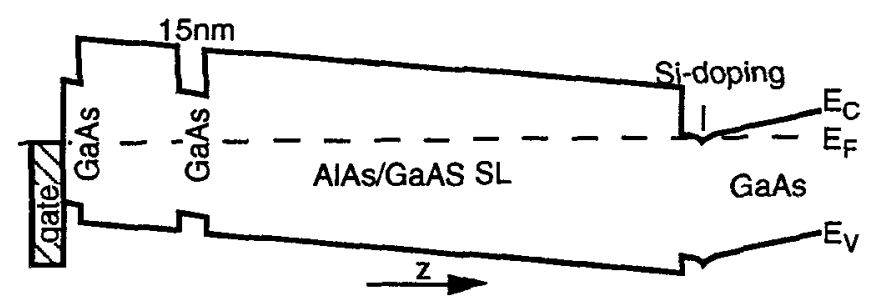

Figure 1: Sketch of the bandstructure of the QW sample with a special design to investigate electric field effects during optical measurements.

This heterostructure is a normally-off device, i.e. it contains no carriers in the quantum well at zero gate bias. Photoluminescence measurements on samples with a homogenous gate yield a positive gate bias of $\mathrm{V}_{\mathrm{th}}=0.7 \mathrm{~V}$ to inject electrons into the QW. Fig. 2 shows luminescence spectra on such a homogenous device recorded with different gate voltages applied.

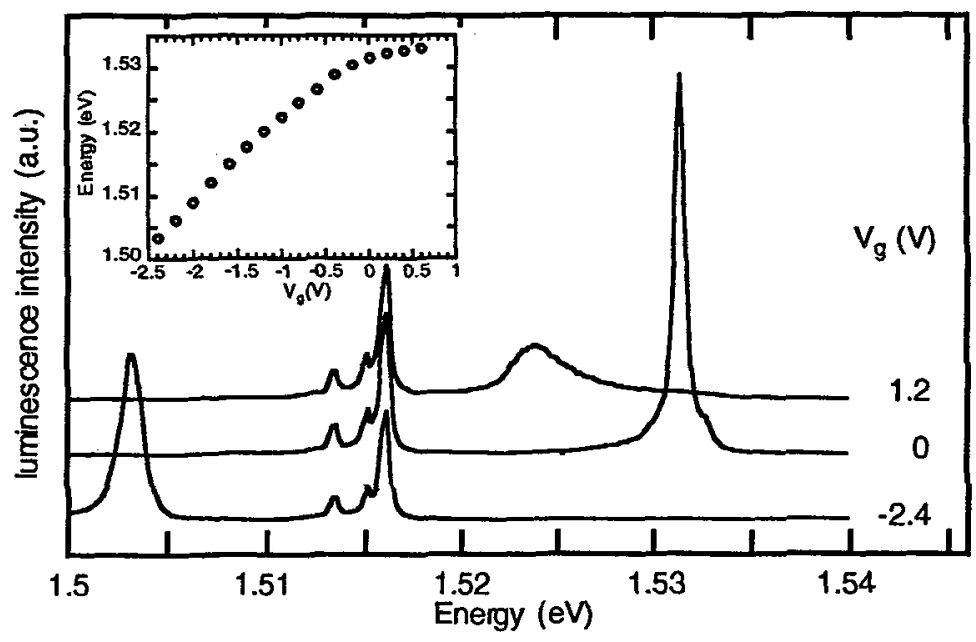

Figure 2: Photoluminescence spectra of the $15 \mathrm{~nm} Q W$ in the optical sample with a homogenous gate on the surface and different gate voltages applied. At $\mathrm{V}_{\mathrm{g}}=1.2 \mathrm{~V}$ electrons are injected into the $\mathrm{QW}$. The signal around $1.515 \mathrm{eV}$ is luminescence of the $\mathrm{GaAs}$ substrate. The inset depicts the energy of the QW-luminescence as function of the gate voltage. 
The energy shifts of the quantum well photoluminescence at gate voltages below $V_{\text {th }}$ reflect the vertical quantum-confined-stark-effect with the same strength already reported in previous studies [12] for a $15 \mathrm{~nm}$ quantum well (inset Fig. 2). At voltages above $V_{t h}=0.7 \mathrm{~V}$ electrons are injected into the quantum well. With gate voltages up to $1.5 \mathrm{~V}$ the electron density can be increased up to $5 \cdot 10^{11} / \mathrm{cm}^{2}$ whereas the leakage current between the gate and the back contact is less than $2 \mu \mathrm{A}$.

\section{Tuneable lateral superlattice}

To induce a lateral superlattice potential at the location of the quantum well, we use a grating gate consisting of $8 \mathrm{~nm}$ semi-transparent $\mathrm{NiCr}$ stripes prepared with a holographic lithography. In contrast to GaAs based samples for FIR or transport measurements, we are using a stacked gate configuration, well known from the silicon technology [13], to control the potential in the gaps between the grating stripes (Fig. 3).

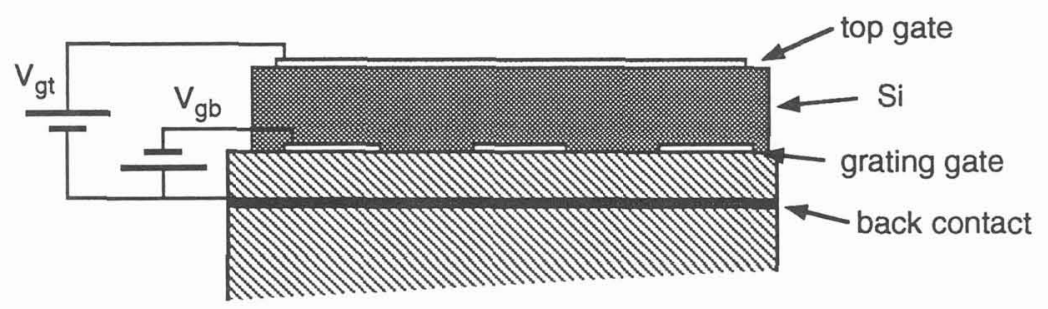

Figure 3: Stacked gate configuration on top of a GaAs/AlAs quantum well sample. The gate voltages of the top and the bottom gate are applied with respect to the back contact beneath the quantum well.

Differently from the silicon samples the spacer between the two gates is made of amorphous Silicon. The spacer has a low conductivity $\left(\sigma=4 \cdot 10^{-11}(\Omega \mathrm{cm})^{-1}\right)$, so that charge accumulation at the interface between the sample structure and the spacer is avoided.

In the following we discuss PL measurements at a stacked gate QW sample in which the bottom grating has a period of $300 \mathrm{~nm}$. Fig. $4 \mathrm{a}$ and $\mathrm{b}$ show the spectra for large positive and negative top gate voltages respectively. In each bottom gate voltages between $-0.3 \mathrm{~V}$ and $1.5 \mathrm{~V}$ are applied. 


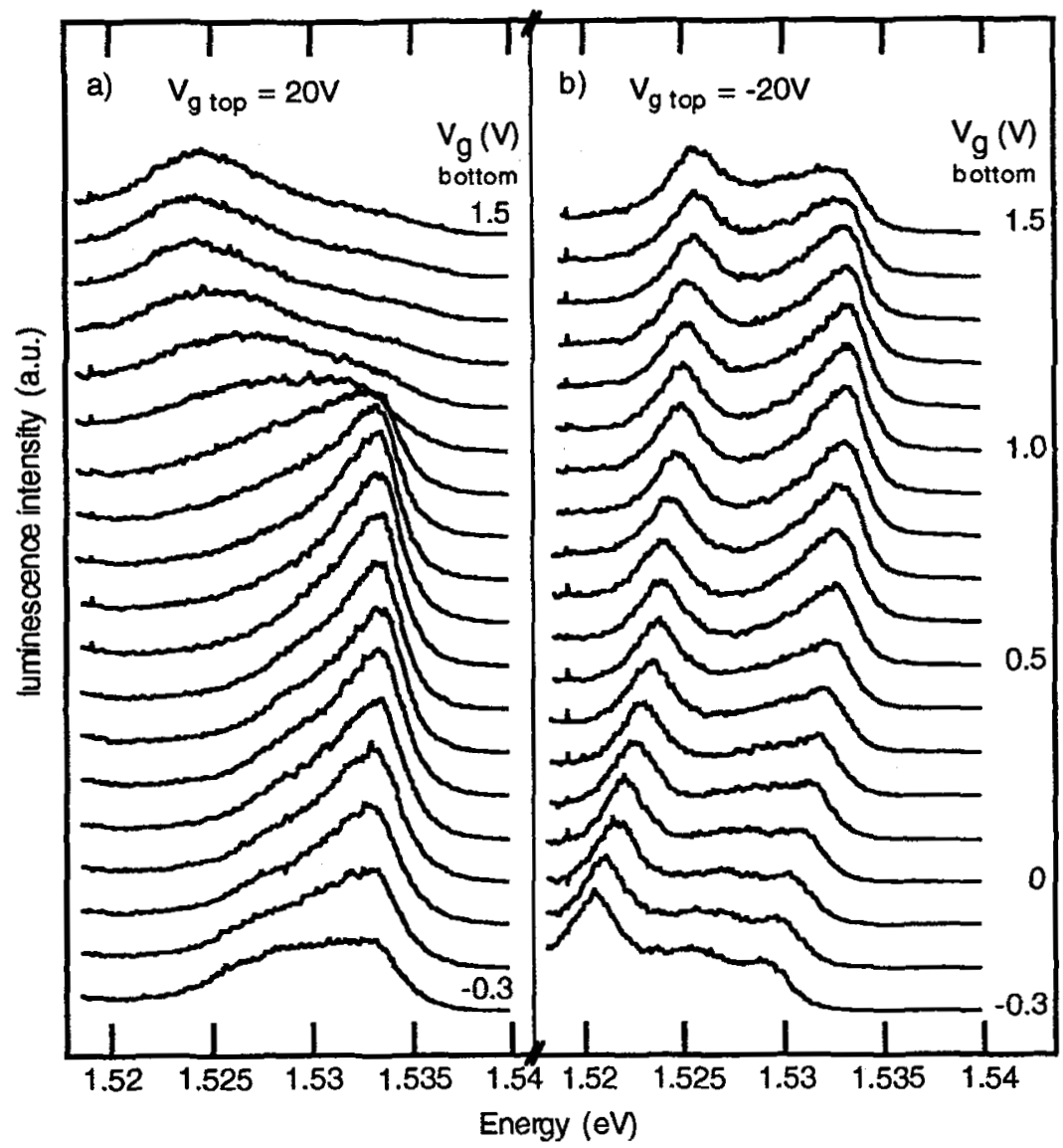

Figure 4: Luminescence spectra of the $15 \mathrm{~nm}$ quantum well with the laser spot on the $300 \mathrm{~nm}$ period double gate structure and a top gate voltage of $20 \mathrm{~V}$ (a) and $-20 \mathrm{~V}$ (b) respectively. The bottom gate voltage is varied in steps of $0.1 \mathrm{~V}$ between $-0.3 \mathrm{~V}$ and $1.5 \mathrm{~V}$.

$\left(\mathrm{T}=2 \mathrm{~K}, \hbar \omega=1.70 \mathrm{eV}\right.$, intensity: $\left.5 \mathrm{~mW}^{\mathrm{m}} / \mathrm{cm}^{2}\right)$.

The PL strongly depends on the applied gate voltage configuration and the PL line shape differs considerably from the PL of the sample with a homogenous gate. The behaviour of the PL may be understood with the aid of the sketches in Fig. 5, visualising the lateral potential configuration for different gate voltage configurations.

For a top gate voltage of $\mathrm{V}_{\mathrm{gt}}=20 \mathrm{~V}$ electrons are induced beneath the gaps of the grating gate. Therefore we observe in Fig. $4 \mathrm{a}$ at $\mathrm{V}_{\mathrm{gt}}=20 \mathrm{~V}$ and $\mathrm{V}_{\mathrm{gb}}>1.2 \mathrm{~V}$ a luminescence spectrum of a nearly homogenous electron gas in the quantum well like sketch d) of Fig. 5 . With decreasing $V_{g b}$ the electron system becomes density modulated (Fig. $5 \mathrm{f}$ ) until at $\mathrm{V}_{\mathrm{gb}} \leq 0.7 \mathrm{~V}$ there are only electrons between the grating stripes forming an array of quantum wires. A significantly differently line shape appears at a top gate voltage of $\mathrm{V}_{\mathrm{gt}}=-20 \mathrm{~V}$ showing a double peak structure over the whole range of bottom voltages. In this case and at $\mathrm{V}_{\mathrm{gb}} \leq 0.7 \mathrm{~V}$ there are no electrons in the $\mathrm{QW}$ and we have a situation as in Fig. 5c). At bottom gate voltages of $\mathrm{V}_{\mathrm{gb}}>0.7 \mathrm{~V}$ we induce electrons in the $\mathrm{QW}$ under the grating gate and we have a situation like in Fig. $5 \mathrm{~g}$ ). 
a)

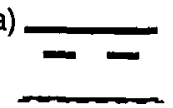

e)

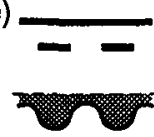

b)

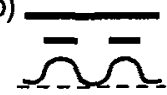

f)

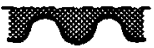

c)

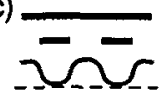

g)

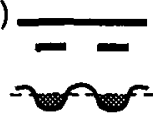

d)

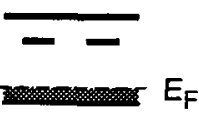

h)

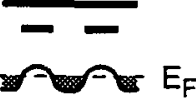

Figure 5: Sketch of the possible potential modulations of the conduction band edge in a quantum well under a double gate structure with respect to the Fermi-energy (doted line). The heavy solid and dashed lines indicate the location of the top gate and the bottom gate, respectively the grey shaded areas mark regions filled with electrons. The sketches a) and d) show the laterally homogenous case without and with electrons, respectively. The pictures b) and c) illustrate a lateral superlattice without electrons, e) and f) show a density modulated electron gas. The pictures $\mathrm{g}$ ) and $\mathrm{h}$ ) represent electron quantum wires induced below the bottom gate and its openings, respectively.

The dominant double peak structure for all voltages in Fig. 4b) leads us to conclude that we have a relatively strong potential modulation and that the two maxima result from recombination involving photo excited carriers from two different regions of the superlattice potential.

In order to identify the origin of the peaks in the luminescence spectra we have performed photoluminescence excitation studies with exemplary results shown in Fig. 6. At a set of gate voltages two excitation spectra are recorded with the detector energy chosen on the low energy shoulder of each of the two distinct PL peaks, respectively. Fig. 6a) demonstrates that at a gate voltage configuration of $\mathrm{V}_{\mathrm{gb}}=1.3 \mathrm{~V}$ and $\mathrm{V}_{\mathrm{gt}}=-20 \mathrm{~V}$ the luminescence excitation spectra even qualitatively differ if they are recorded with different detector energies. The line shape of the excitation spectrum recorded with the detector energy at $1.523 \mathrm{eV}$ indicates that the signal originates from the areas of the $\mathrm{QW}$ beneath the gate strips that are occupied with electrons. A Burstein-Moss-shift [14] of $10 \mathrm{meV}$ between the PL and the PLE signal is observed and the PLE onset exhibits no excitonic maximum. In contrast the PLE spectrum recorded with the detector positioned at the high energy luminescence peak $(1.531 \mathrm{eV})$ is not shifted with respect to the detector position and shows two excitonic peaks at $1.535 \mathrm{eV}$ and $1.542 \mathrm{eV}$ very similar to the heavy and light hole exciton peaks of the absorption spectrum in a homogeneous reference sample with an empty well. We thus infer that the two luminescence peaks observed in this configuration stem from regions of the sample that are occupied and unoccupied, respectively. The occupied regions form an array of quasi-one-dimensional electron channels beneath the metal stripes of the bottom gate.

At $V_{\mathrm{gb}}=0 \mathrm{~V}$ and $\mathrm{V}_{\mathrm{gt}}=-20 \mathrm{~V}$ we do not expect electrons to occupy the QW. Surprisingly, we also observe two peaks in the PL spectrum at this gate voltage configuration as shown in Fig. 6b). However in this case, both the corresponding PLE spectra show no Burstein-Moss-shift, in agreement with our assumption of an empty QW. Furthermore, at both detector energies we do not observe excitonic peaks in the PLE spectra. This indicates that a strong lateral potential modulation quenches the exciton peaks in the excitation spectra. Indeed an estimate of the lateral fields generated at this gate voltage shows that the fields are sufficiently high to prevent formation of excitonic states. 


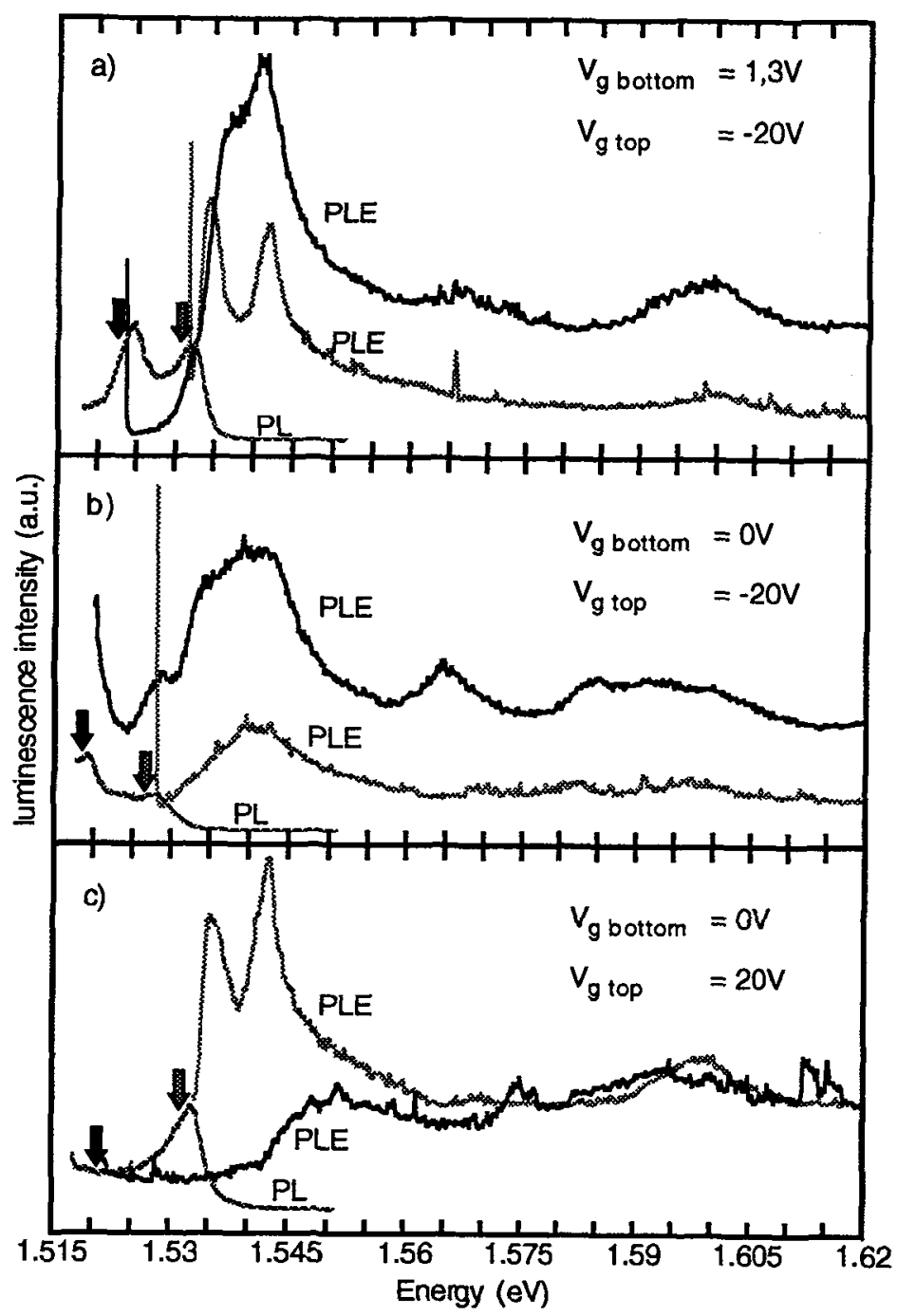

Figure 6: Photoluminescence (PL) and photoluminescence excitation (PLE) spectra of the quantum well under the double gate structure with different gate voltages applied. The PLE spectra are recorded with detector positions on the low energy tail of the corresponding PL signal as indicated by the arrows.

At the gate biases $\mathrm{V}_{\mathrm{gb}}=0 \mathrm{~V}$ and $\mathrm{V}_{\mathrm{gt}}=20 \mathrm{~V}$ of Fig. $6 \mathrm{c}$ ) we observe one PL peak with a wide low energy tail. Similar to spectra in Fig. 6a) the excitation spectra recorded at the PL maximum and in the low energy tail again show excitonic behaviour and a Burstein-Moss-shift, respectively, indicating that again a electron wire array is formed. In this case the quantum wires are induced by the positively biased top gate beneath the gaps of the bottom gate.

In the results presented above the excitation intensity was kept comparatively low at about $5 \mathrm{~mW} / \mathrm{cm}^{2}$. With increasing excitation intensity we expect an existing potential modulation to be screened due to the photo excited carriers and therefore to observe a decrease of the effect of the potential modulation [15]. Representative results of such intensity dependent luminescence studies are shown in Fig. 7. At positive 
voltages on both gates we expect a rather homogenous electron gas in the QW resulting in a luminescence signal that depends only little on excitation intensity as in Fig. 7a). The broadening at higher intensity originates from the photo generated carriers. The substrate signal around $1.515 \mathrm{eV}$ saturates at higher intensities. At $\mathrm{V}_{\mathrm{gb}}=1.3 \mathrm{~V}$ and $\mathrm{V}_{\mathrm{gt}}=-20 \mathrm{~V}$ we observe the double peak structure for low intensities reflecting a strong potential modulation. With increasing power this double peak smears out and we observe a spectrum similar to the one of a homogenous QW occupied with electrons. Obviously the increasing number of photo induced carriers screen the potential modulation. The behaviour is therefore as expected for a lateral potential modulation superlattice and supports the interpretation of the previous PL and PLE spectra.

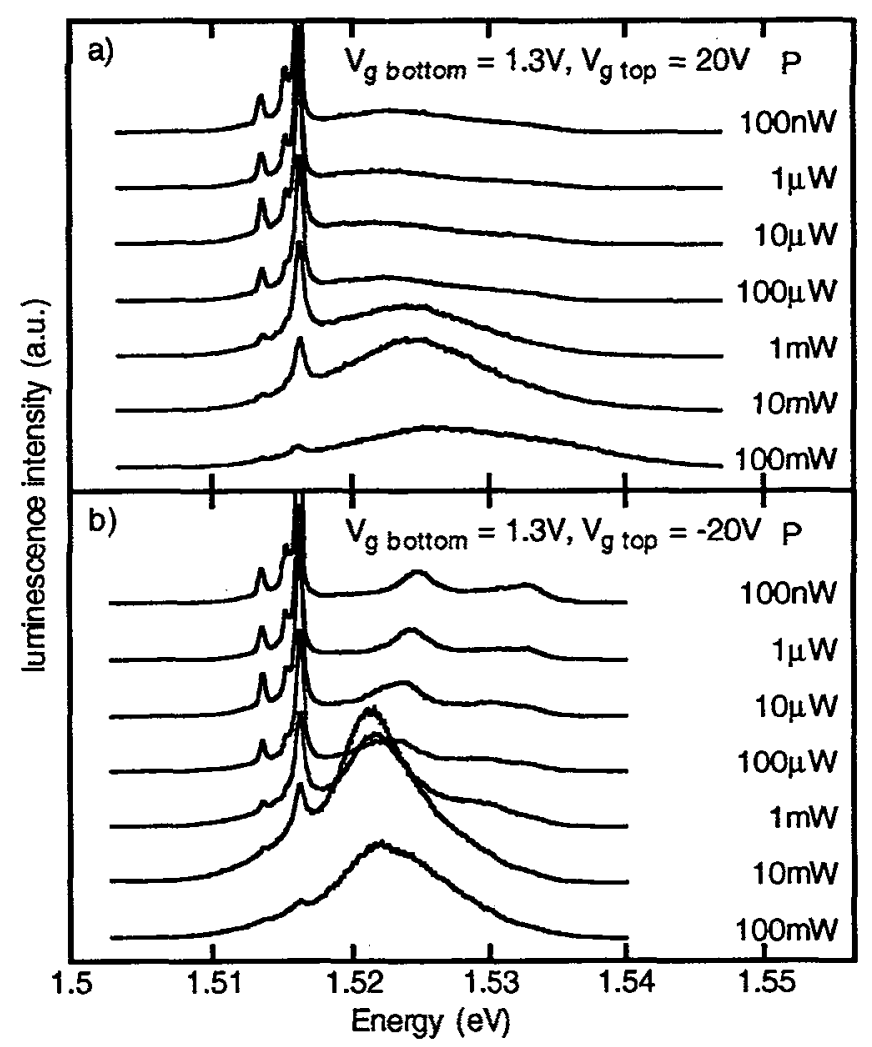

Figure 7: Power dependent luminescence of the $15 \mathrm{~nm}$ quantum well under the double gate structure. Part a) shows the homogenous case, part b) with a lateral potential modulation. $\left(\mathrm{T}=2 \mathrm{~K}, \hbar \omega=1.70 \mathrm{eV}\right.$, laser spot: $\left.50 \times 50 \mu \mathrm{m}^{2}\right)$.

In view of the strong potential modulation two observations are surprising. One is that luminescence is seen for two distinct regions of the superlattice. This implies that under illumination holes as minority carriers are present both in the minima and the maxima of the superlattice potential in spite of the lateral electric field. The second is that spatial indirect transitions as reported by Weiner [2], Hirler [15] and Fritze [16] are not seen though periods in these etched devices are about the same as in ours. 


\section{Conclusion}

With a new stacked gate technology and a special MBE-structure we are able to study the effect of a field- effect-induced lateral potential modulation on the optical response of a GaAs quantum well. The optical properties of the quantum well depend strongly on the potential modulation. We have shown with the help of luminescence and excitation spectroscopy that we are able to induce an array of quasi-onedimensional electron wires under the stripes of the grating gate as well as under the top gate electrode. Because of the tuneable potential transitions between electron systems of different dimensions can be observed.

We acknowledge financial support by the Deutsche Forschungsgemeinschaft via SFB 348.

\section{References}

[1] M. Kohl, D. Heitmann, P. Grambow, and K. Ploog, Phys. Rev. Lett. 63 (1989) 2124.

[2] J.S Weiner, G. Danan, A. Pinczuk, J. Valladares, L.N. Pfeiffer, and K. West, Phys. Rev. Lett. 63 (1989) 1641.

[3] F. Hirler, R. Strenz, R. Küchler, G. Abstreiter, G. Böhm, J. Smoliner, G. Tränkle and G. Weimann, Semicond. Sci. Technol. 8 (1993) 617.

[4] K. Brunner, U. Bockelmann, G. Abstreiter, M. Walther, G. Böhm, G. Tränkle and G. Weimann, Phys. Rev. Lett. 69 (1992) 3216.

[5] E. Kapon, D. M. Hwang and R. Bhat, Phys. Rev. Lett. 63 (1989) 430.

[6] K. Kash, J. M. Worlock, M. D. Sturge, P. Grabbe, J. P. Harbison, A. Scherer and P. S. D. Lin, Appl. Phys. Lett. 53 (1988) 782.

[7] W. Hansen, M. Horst, J.P. Kotthaus, U. Merkt, Ch. Sikorski and K. Ploog, Phys. Rev. Lett. 58 (1987) 2586.

[8] R.W. Winkler, J.P. Kotthaus and K. Ploog, Phys. Rev. Lett. 62 (1989) 1177.

[9] D.A.B. Miller, D.S. Chemla, T.C. Damen, A.C. Gossard, W. Wiegmann, T.H. Wood and C.A. Burrus, Phys. Rev. Lett. 53 (1984) 2173.

[10] C. Delalande, G. Bastard, J. Orgonasi, J.A. Brum, H.W. Liu, M.Voos, G. Weimann and W. Schlapp, Phys. Rev. Lett. 59 (1987) 2690.

[11] E.E. Mendez, F. Agullo-Rueda and J.M. Hong, Phys. Rev. Lett. 60 (1988) 2426.

[12] E.E. Mendez and F. Agull6-Rueda, Journal of Luminescence 44 (1989) 223.

[13] J. Alsmeier, E. Batke and J.P. Kotthaus, Phys. Rev. B 41 (1990) 1699.

[14] S. Schmitt-Rink, D.S. Chemla and D.A.B. Miller, Advances in Physics 38 (1989) 89.

[15] F. Hirler, R. Küchler, R. Strenz, G. Abstreiter, G. Böhm, J. Smoliner, G. Tränkle and G. Weimann, Surf. Sci. 263 (1992) 536.

[16] M. Fritze, W. Chen, A.V. Nurmikko and P. Hawrylak, "Fermi-edge Singularities in InGaAs and GaAs Quantum Wires", proceedings of the EP2DS-10, to be published. 